\title{
Effect of Laser Annealing on Optical Properties of Ion-Implanted ZnO Nanorods
}

\author{
Tetsuya Shimogaki $^{* 1}$, M. Higashihata ${ }^{* 2}$, D.Nakamura ${ }^{* 2}$, T. Asano ${ }^{* 2}$, T. Okada ${ }^{* 2}$ \\ ${ }^{*}$ Graduate School of Information Science and Electrical Engineering, Kyushu Univ., 744 Motooka, \\ Nishi-ku, Fukuoka 819-0395, Japan \\ E-mail:shimogaki@laserlab.ees.kyushu-u.ac.jp \\ ${ }^{* 2}$ Faculty of Information Science and Electrical Engineering, Kyushu Univ., 744 Motooka, Nishi- \\ ku, Fukuoka 819-0395, Japan
}

\begin{abstract}
We report on the demonstration of the effectiveness of nanosecond laser annealing on optical properties of phosphorus ion $\left(\mathrm{P}^{+}\right)$-implanted $\mathrm{ZnO}$ nanorods (NRs). Vertically-alligned $\mathrm{ZnO} \mathrm{NRs}$ have been synthesized by nanoparticle-assisted pulsed laser deposition (NAPLD) on $c$-plane sapphire substrates. The pre-laser annealing was performed with the third harmonic (355 nm) of a Qwitched Nd:YAG laser at a fluence of $100 \mathrm{~mJ} / \mathrm{cm}^{2}$ for improving optical property and weakening $n$ type conduction of as-grown $\mathrm{ZnO}$ NRs. $\mathrm{P}^{+}$-ions were implanted into the $\mathrm{ZnO}$ NRs followed by the post-laser annealing in the same way as the pre-laser annealing. The top part of the ZnO NRs were melted and recrystallized by laser annealing. The transitions of properties were investigated by the scanning electron microscope, the room temperature photoluminescence spectra, X-ray diffraction patterns, and $I-V$ measurement. As a result, it turned out that laser annealing has potentiality for improving optical property and compensating donor-vacancies without reducing crystalline quality. Moreover, the effectiveness of post-laser annealing on recovering ion-implanted $\mathrm{ZnO}$ layer and activating acceptors was demonstrated. It is promising that straightforward and stable method of fabricating $\mathrm{ZnO}$-based UV-LED is established by applications of nanosecond laser annealing process. DOI:10.2961/jlmn.2013.01.0015
\end{abstract}

Keywords: zinc oxide, single-crystal, nanostructure, laser annealing, ion implantation

\section{Introduction}

Zinc oxide $(\mathrm{ZnO})$, is rich in natural resources, has a wide band-gap energy of approximately $3.37 \mathrm{eV}$ and a large exciton binding energy of $60 \mathrm{meV}$ that larger than the thermal energy of $26 \mathrm{meV}$ at room temperature. These can ensure an efficient exciton emission in near ultraviolet spectral region. This is why $\mathrm{ZnO}$ is considered the most attractive material for optoelectronic application in particular near ultraviolet light emitting diode (UV-LED). In addition, since $\mathrm{ZnO}$ has a strong tendency for self-organized growth, a variety of $\mathrm{ZnO}$ single-crystal nanostructures have been synthesized.

However, the construction of homo $p-n$ junction is still problematic, and especially the method for the realization of the stable $p$-type $\mathrm{ZnO}$ has not been well established. This is due to the self-compensation effect of $\mathrm{ZnO}$ which characterizes $\mathrm{ZnO}$ native $n$-type conduction. ${ }^{[1]}$ Many groups have been studying for fabricating $p$-type $\mathrm{ZnO}$ crystals by different means of growth methods, including carbo-thermal method, pulsed-laser deposition, hydro-thermal method and so on, with different acceptor dopants like nitrogen, phosphorous, antimony, or co-doping of lithium and nickel and so on. ${ }^{[2-7]}$ Among them, ion implantation is a straightforward method of controlling the majority carrier concentration. X. W. Sun et al. reported the fabrication of $p$ - ZnO by means of phosphorus ion $\left(\mathrm{P}^{+}\right)$-implantation and the construction of homo $p-n$ junction in the $\mathrm{ZnO}$ nanowires. ${ }^{[4]}$ In the case of ion implantation, the annealing process is absolutely necessary for the recovery of the crys- tals and optical property, since the ion implantation causes damage to crystals and large amount of defects bring degradation of optical properties.

In this study, we have investigated the effects of the nanosecond laser annealing on morphological and optical properties of $\mathrm{ZnO}$ nanorods (NRs). The experimental procedures are displayed briefly in Fig. 1. The pre-laser annealing (pre-LA) made ZnO NRs' crystalline quality better and prepared for ion implantation by compensating defects which is an origin of $n$-type conduction. Then, phosphorus ions $\left(\mathrm{P}^{+}\right)$were implanted in pre-LA ZnO NRs. Finally, the

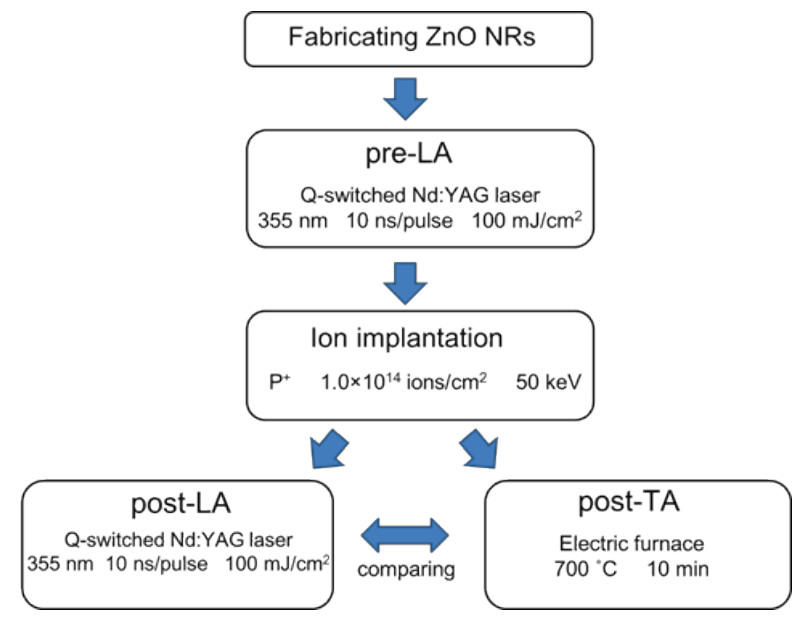

Fig. 1 Flowchart of experimental procedures. 
post-laser annealing (post-LA) recovered the crystal structure and room temperature photoluminescence (RT-PL) property of the $\mathrm{P}^{+}$-implanted $\mathrm{ZnO}$ NRs. In addition, it was compared with RT-PL spectrum of ZnO NRs after postthermal annealing (post-TA) by an electric furnace. We report on the effectiveness of nanosecond laser annealing for fabricating $p$-type nanostructured $\mathrm{ZnO}$ by means of ion implantation.

\section{Experiment}

\subsection{ZnO NRs preparation}

Vertically-aligned $\mathrm{ZnO}$ NRs used in this study were synthesized by nano particle assisted pulsed laser deposition (NAPLD). NAPLD does not require any catalysts and high-vacuum environment. Furthermore, various $\mathrm{ZnO}$ nanostructures can be grown on the many substrates by controlling experimental conditions. ${ }^{[8-11]}$ Densely-packed $\mathrm{ZnO} \mathrm{NRs}$, which is adequate to ion implantation, was adopted in this study.

A sintered pure $\mathrm{ZnO}$ target placed in a vacuum chamber was ablated for $25 \mathrm{~min}$ with the third harmonic of a Qswitched Nd:YAG laser (Quanta-Ray, GCR-290, Spectra Physics) at $355 \mathrm{~nm}$ with a repetition rate of $10 \mathrm{~Hz}$ and a fluence of approximately $1.5 \mathrm{~J} / \mathrm{cm}^{2}$. The pressure in the chamber and the $\mathrm{O}_{2}$ gas influx were kept at $667 \mathrm{~Pa}$ and 20 $\mathrm{cm}^{3} / \mathrm{min}$, respectively. ZnO NRs were grown on thermal annealed $\left(1000{ }^{\circ} \mathrm{C}\right.$, for $\left.120 \mathrm{~min}\right) c$-cut sapphire substrate placed on a $\mathrm{SiC}$ plate heated at $700{ }^{\circ} \mathrm{C}$ faced the $\mathrm{ZnO}$ target at a distance of $40 \mathrm{~mm}$.

Scanning electron microscope (SEM) images of the asgrown $\mathrm{ZnO}$ NRs are shown in Fig. 2 (a) and (b). Figure 2 (a) and the upper right inset in Fig. 2 (a) display the view tilted by $45^{\circ}$ and the top view, respectively. ZnO NRs with a diameter of approximately $500 \mathrm{~nm}$ are densely grown on the whole surface of substrate. In addition, the height of $\mathrm{ZnO}$ NRs is approximately $3 \mu \mathrm{m}$ as shown in Fig. 2 (b).

\subsection{Pre-laser annealing}

The pre-LA was performed in air with the Q-switched $\mathrm{Nd}$ :YAG laser at $355 \mathrm{~nm}$ and a fluence of $100 \mathrm{~mJ} / \mathrm{cm}^{2}$ by $10 \mathrm{~ns} /$ pulse. The laser beam was irradiated on the surface of $\mathrm{ZnO}$ NRs while the position was adjusted by precision $\mathrm{XY}$ stage so that the number of irradiation should be 1 pulse/location. ${ }^{[12]}$

Figure 3 (a) displays a SEM image of pre-LA ZnO NRs. The top part of $\mathrm{ZnO}$ NRs were melted and recrystallized. The X-ray diffraction (XRD) patterns in Fig. 3 (b)

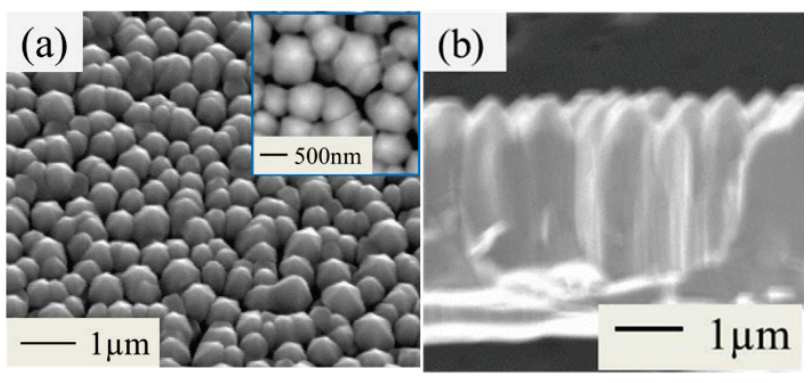

Fig. 2 (a) SEM images of as-grown $\mathrm{ZnO}$ NRs viewed from top and tilted $45^{\circ}$. (b) A side-view SEM image of as-grown $\mathrm{ZnO}$ NRs.

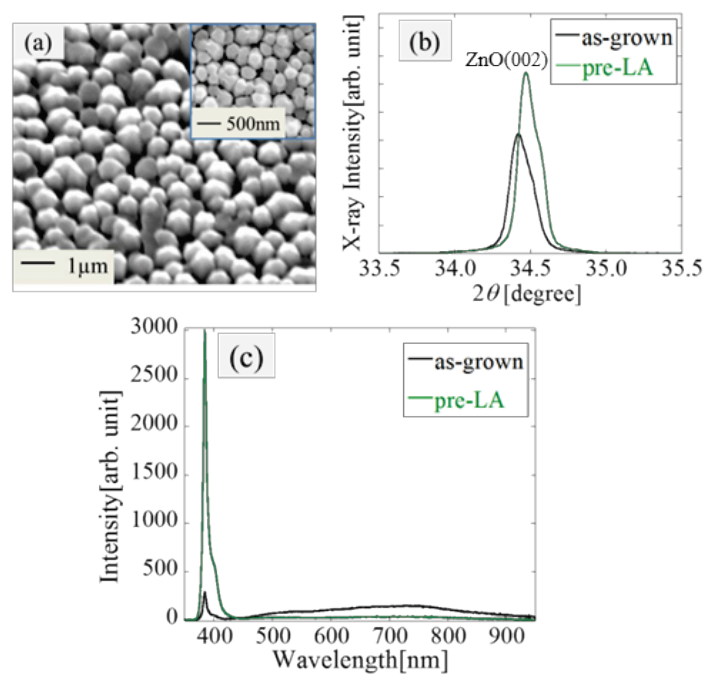

Fig. 3 (a) A SEM image of pre-LA ZnO NRs viewed from top and tilted $45^{\circ}$. (b) XRD patterns of asgrown and pre-LA ZnO NRs. (c) RT-PL spectra of as-grown and pre-LA ZnO NRs.

show peaks appearing at $2 \theta \sim 34.5$, which correspond to the (002) direction of the wurtzite hexagonal structure of as-grown $\mathrm{ZnO} \mathrm{NRs}$ and pre-LA ZnO NRs. In other area, no significant peak was observed except at $2 \theta \sim 72^{\circ}$, which correspond to (004) direction of that. It can be seen that crystalline quality was not reduced by melting process of the pre-LA. It is expected that the shift of diffraction peak is attributed to lattice strain relaxation effect of the laser annealing. Figure 3 (c) displays RT-PL spectra of the preLA and the as-grown $\mathrm{ZnO}$ NRs measured with a spectrometer (C10027-01, Hamamatsu Photonics K.K.) under the excitation at $325 \mathrm{~nm}$ using a He-Cd laser (IK3301 R-G, KIMMON KOHA Co., Ltd.). The pre-LA ZnO NRs have a sharp near band edge (NBE) emission compared with asgrown $\mathrm{ZnO}$ NRs. In addition, visible emission caused by natural defects in $\mathrm{ZnO}$ crystals was decreased to $25 \%$. ${ }^{[13]}$ These facts mean that crystalline quality was improved, and natural defects in $\mathrm{ZnO}$ crystals which cause $n$-type conduction were compensated.

\subsection{Ion implantation}

A half region of as-grown $\mathrm{ZnO} \mathrm{NRs}$ grown on the substrate placed in the vacuum chamber was implanted at room temperature with $\mathrm{P}^{+}$by an ion implantation equipment (UR-200, ULVAC). The acceleration voltage and the dosage were set at $50 \mathrm{keV}$ and $1.0 \times 10^{14}$ ions $/ \mathrm{cm}^{2}$, respectively. The implantation angle was $7^{\circ}$ to reduce channeling effects.

In SEM observation, no differences exist between the surface of as-grown and pre-LA ZnO NRs in appearance (not shown). The XRD pattern of the as-implanted $\mathrm{ZnO}$ NRs is displayed in Fig. 4 (a) with the red line. The XRD pattern of pre-LA ZnO NRs is also shown in Fig. 4 (a) for comparison. For this reason, it was found that crystalline structures of the surface were broken by ion implantation. Penetration depth of implanted $\mathrm{P}^{+}$was estimated to approximately $200 \mathrm{~nm}$ by the TRIM (the Transport of Ions in Matter) code. Since X-rays infiltrate over $200 \mathrm{~nm}$, the $\mathrm{XRD}$ peak remained to be measured. RT-PL property is 


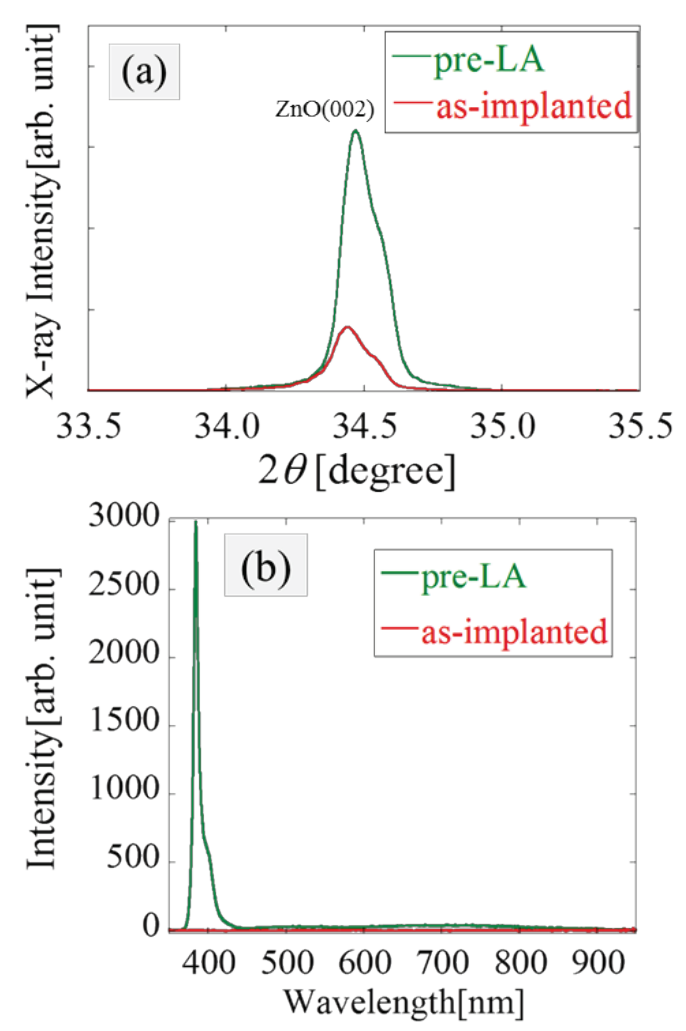

Fig. 4 (a) XRD patterns of pre-LA and as-implanted ZnO NRs. (b) RT-PL spectra of pre-LA and asimplanted $\mathrm{ZnO}$ NRs.

displayed in Fig. 4 (b). No luminescence was observed throughout all wavelength regions between $350 \mathrm{~nm}$ and $950 \mathrm{~nm}$. For this reason and the fact that $\mathrm{ZnO}$ have a slight transmittance toward $\mathrm{He}-\mathrm{Cd}$ laser, it is speculated that many defects were created in the crystal structure by ion implantation. ${ }^{[14]}$

\subsection{Post-laser annealing}

Next, the post-LA was performed at a fluence of 100 $\mathrm{mJ} / \mathrm{cm}^{2}$ with the Q-switched Nd:YAG laser. The laser beam was irradiated on the surface of the $\mathrm{P}^{+}$-implanted region with a single irradiation condition by the same way as pre-LA.

Figure 5 (a) displays a SEM image of the $\mathrm{P}^{+}$-implanted $\mathrm{ZnO}$ NRs followed by the post-LA. As can be seen from Fig. 5 (a), top part of $\mathrm{ZnO}$ NRs was melted more than the one after pre-LA. The XRD pattern of the post-LA ZnO NRs is shown in Fig. 5 (b), along with that of as-implanted $\mathrm{ZnO}$ NRs. The crystalline quality is recovered. That is, damaged $\mathrm{ZnO}$ crystals were recrystallized by the post-LA. The RT-PL property of ZnO NRs after post-LA is displayed in Fig. 5 (c), where compares it with post -TA ZnO NRs by the electric furnace as a reference. In the case of the post-LA, only NBE emission was recovered. It can be seen that defects of $\mathrm{P}^{+}$-implanted $\mathrm{ZnO} \mathrm{NRs}$ are fully compensated by the post-LA. On the other hand, $\mathrm{P}^{+}$-implanted $\mathrm{ZnO} \mathrm{NRs}$ followed by the post-thermal annealing (postTA) had little NBE emission and strong visible emission caused by new defects in $\mathrm{ZnO}$ NRs. It was performed with the electric furnace at $700{ }^{\circ} \mathrm{C}$ for $10 \mathrm{~min}$. These are attributed to the fact that thermal annealing is a process by
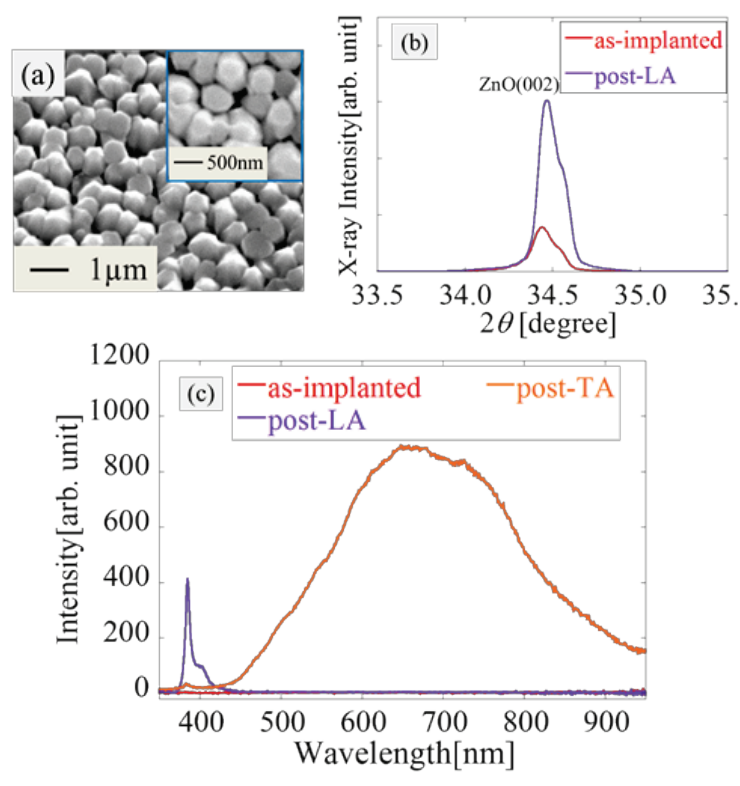

Fig. 5 (a) A SEM image of $\mathrm{P}^{+}$-implanted $\mathrm{ZnO}$ NRs followed by the post-LA. (b) XRD patterns of asimplanted and post-LA ZnO NRs. (c) RT-PL spectra of as-implanted, post-LA, and post-TA $\mathrm{ZnO} \mathrm{NRs}$ by the electric furnace.

atomic motion. Accordingly, acceptors diffused excessively and many $\mathrm{Zn}$ and $\mathrm{O}$-sites remained as defects.

Consequently, defects of $\mathrm{P}^{+}$-implanted $\mathrm{ZnO}$ NRs were compensated insufficiently by post-TA. As stated above, the effectiveness of the laser annealing for recovering optical properties of $\mathrm{P}^{+}$-implanted $\mathrm{ZnO} \mathrm{NRs}$ has been demonstrated.

\section{I-V measurement}

Figure 6 displays the $I-V$ characteristics of $\mathrm{P}^{+}$-implanted $\mathrm{ZnO}$ NRs followed by the post-LA. This $I-V$ characteristics were measured by forming ohmic contact on both $p$ type and $n$-type regions. Metal-semiconductor ohmic contacts were realized by using a tungsten probe and golddeposited probe as shown in lower left inset of Fig. $6 .{ }^{[15]}$ This achievement of ohmic contact is proofed by upper left inset of Fig. 6, which displays linear $I-V$ characteristics measured between two different areas only on unimplanted $\mathrm{ZnO} \mathrm{NRs}$ or on the $\mathrm{P}^{+}$-implanted $\mathrm{ZnO}$ NRs followed by the post-LA.

A good rectifying characteristic was observed in Fig. 6. The threshold voltage in the positive bias was approximately $6 \mathrm{~V}$. Since as-grown $\mathrm{ZnO}$ NRs have $n$-type conduction, the rectifying characteristic indicates that the $p$-type conduction is realized in the $\mathrm{P}^{+}$-implanted $\mathrm{ZnO}$ NRs followed by the post-LA. In addition, linear $I-V$ characteristics were observed in the case of post-TA (not shown).

\section{Conclusion}

In summary, we have demonstrated the effectiveness of the nanosecond laser annealing on $\mathrm{ZnO}$ NRs. The pre-LA improved optical property of as-grown $\mathrm{ZnO}$ NRs without reduction of their crystalline quality. We speculated that $n$ type conduction of as-grown $\mathrm{ZnO}$ NRs was reduced. The post-LA recovered the crystals and optical property of $\mathrm{P}^{+}$implanted $\mathrm{ZnO}$ NRs. In particular, dramatic recovery of 


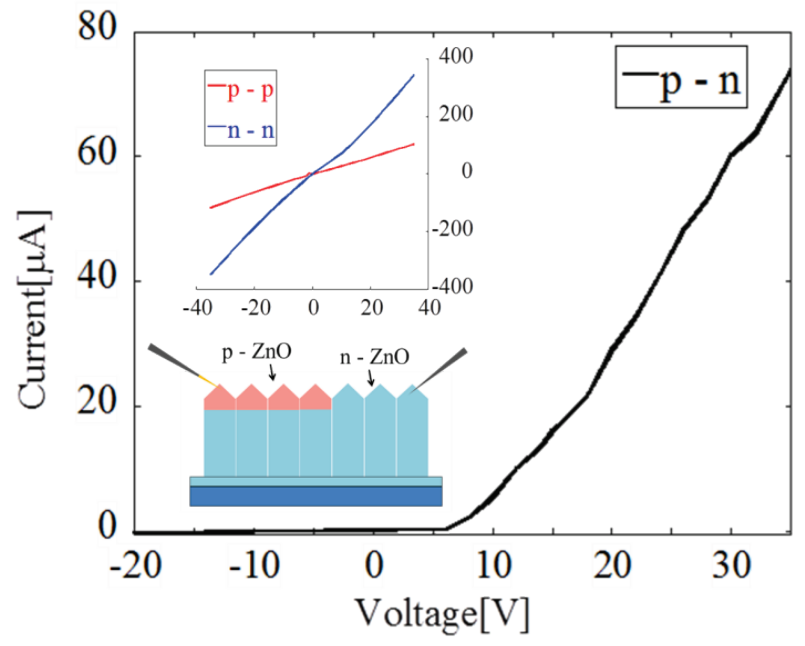

Fig. $6 I-V$ characteristics of $\mathrm{P}^{+}$-implanted $\mathrm{ZnO}$ NRs followed by the post-LA. The upper left inset displays $I-V$ characteristics at $p-p$ and $n-n$ areas. The lower left inset displays a schematic of contacting probe electrode.

optical property has been achieved by the post-LA, compared with the post-TA. As the result, the fabrication of the $p-n$ homo junction along $\mathrm{ZnO}$ NRs has been demonstrated using $\mathrm{P}^{+}$-ion implantation and nanosecond laser annealing. Our research provided a strong possibility of realization of constructing a new and simple fabrication method of $\mathrm{ZnO}$ based UV-LED by ion implantation.

\section{Acknowledgments and Appendixes}

The authors would like to thank Mr. Takayuki Takao for his supports in the ion implantation experiment. This work was supported in part by the Program under Special Coordination Funds for Promoting Science and Technology from Japan Science and Technology Agency (JST) and a Grant-in-Aid for Scientific Research from the Japan Society for the Promotion of Science (JSPS, No. 24656053).

\section{References}

[1] K. G. Saw, K. Ibrahim, Y. T. Lim and M. K. Chai, Thin Solid Films, 50, 2879 (2007).

[2] K. Nakahara, S. Akasaka, H. Yuji, K. Tamura, T. Fujii, Y. Nishimoto, D. Takamizu, A. Sasaki, T. Tanabe, H. Takasu, H. Amaike, T. Onuma, S. F. Chichibu, A. Tsukazaki, A. Ohtomo and M. Kawasaki, Appl. Phys. Lett. 97, 013501 (2010).

[3] M. T. Chen, M. P. Lu, Y. J. Wu, J. Song, C. Y. Lee, M. Y. Lu, Y. C. Chang, L. J. Chou, Z. L. Wang and L. J. Chen, Nano Lett. 10, 4387 (2010).

[4] X. W. Sun, B. Ling, J. L. Zhao, S. T. Tan, Y. Yang, Y. Q. Shen, Z. L. Dong and X. C. Li, Appl. Phys. Lett. 95, 133124 (2009).

[5] V. Vaithianathan, B. T. Lee and S. S. Kim, J. Appl. Phys. 98, 043519 (2005).

[6] T. Aoki, Y. Shimizu, A. Miyake, A. Nakamura, Y. Nakanishi and Y. Hatanaka, Phys. Status Solidi B, 229, 911 (2002).

[7] E. S. Kumar, J. Chatterjee, N. Rama, N. DasGupta and M. S. R. Rao, Appl. Mater. Interfaces, 3, 1974 (2011).
[8] D. Nakamura, A. Kumeda, K. Toya, K. Okazaki, K. Kubo, K. Tsuta, M. Higashihata and T. Okada, Proc. TENCON 2010, 990 (2011).

[9] K. Sakai, S. Oyama, K.Noguchi, A.Fukuyama, T.Ikari and T. Okada, Physica E 40, 2489 (2008).

[10] Y. Nakata, B. H. Agung and T. Okada, Appl. Phys. A, 79, 1417 (2004).

[11] D. Nakamura, T. Okada and K. Sakai, Proc. SPIE, 7996, 79960E (2010).

[12] J. Maeng, S. Heo, G. Jo, M. Choe, S. Kim, H. Hwang and T. Lee, Nanotechnology, 20, 095203 (2009).

[13] A. Janotti and C. G. V. Walle, Appl. Phys. Lett. 87, 122102 (2005).

[14] Z. Q. Chen, A. Kawasuso, Y. Xu, H. Naramoto H. Yan, X. L. Yuan, T. Sekiguchi, R. Suzuki and T. Ohdaira, J. Appl. Phys. Lett. 97, 013528 (2005).

[15] L. J. Brillson and Y. Lu, J. Appl. Phys. 109, 121301 (2011).

(Received: June 08, 2012, Accepted: January 16, 2013) 